Conclusions Most patients with staphylococcal infections can be treated with vancomycin, which also contributes to cost reduction. A Bayesian approach shows better pharmacodynamic results than conventional dosing, with a $90 \%$ of patients successfully treated in a real setting.

No conflict of interest.

\section{PHC-005 BLOOD LEVELS OF IMMUNOSUPPRESSANT DRUGS IN PATIENTS WITH CYSTIC FIBROSIS AFTER LUNG TRANSPLANTATION}

doi:10.1136/ejhpharm-2013-000276.350

J Ruiz, A García, P Pérez, M Cueto, R Marqués, JL Poveda. Hospital Universitario La Fe, Pharmacy, Valencia, Spain

Background Patients with Cystic Fibrosis (CF) can absorb oral drugs differently, which could be translated into reduced blood levels of immunosuppressant drugs in transplant patients.

Purpose To evaluate the blood levels of immunosuppressant drugs in patients with CF after lung transplantation during the first months of oral treatment and their effect on the development of acute rejection (AR) and renal failure (RF).

Materials and Methods Retrospective observational study (study period: April 2008 to October 2012). Tacrolimus and mycophenolic acid blood levels of lung transplant patients were collected during the first three months of oral treatment. Blood levels were corrected by dose and body weight [(Concentration/(dose/weight)) (Concentration $=\mathrm{ng} / \mathrm{mL}$ for tacrolimus and $\mathrm{mcg} / \mathrm{mL}$ for mycophenolic acid; dose $=\mathrm{mg} / \mathrm{kg} / 24 \mathrm{~h}$; weight $=\mathrm{kg}$ )]. The primary outcome was to compare immunosuppressant levels between patients with CF and other transplant patients (control group). The incidence of AR and RF (Chi-square test) and overall survival (KaplanMeier method) were calculated in both groups.

Results Sample size 49 patients $(69.0 \%$ male, mean age $=45.2$ $(\mathrm{SD}=16.2)$ years), of which $27.0 \%$ were CF patients. Immunosuppressant blood levels were lower in the CF group compared with the control group [mean(SD)): Tacrolimus: month 1: 67.6(34.9) vs. 105.6(58.2)*; month 2: $64.9(36.5)$ vs. $140.2(106.3)^{*}$, month 3: $97.0(76.6)$ vs. $129.8(128.2)$; Mycophenolic acid: month 1: 0.05(0.03) vs. $0.09(0.14)^{*}$, month 2 : $0.09(0.08)$ vs. $0.09(0.04)$ month 3 : $0.20(0.17)$ vs. $0.16(0.14)(* p<0.05$, Wilcoxon-T test)]. The incidence of AR was higher in the CF group (53.8\% vs. $47.2 \%, p=0.84$ ), while the incidence of RF was higher in the control group $(27.8 \%$ vs. 23. $0 \%, p=0.74)$. Overall survival after transplantation was higher in the CF group (51.1 vs. 39.1 months, $\mathrm{p}=0.08$ ).

Conclusions Patients with CF have lower immunosuppressant levels than the control group. However, there were no significant differences in the incidence of AR, the development of RF or in overall survival after transplantation between the two groups.

No conflict of interest.

\section{PHC-006 CONCOMICANT DRUGS AS A RISK FACTOR FOR THE APPEARANCE OF ADVERSE EVENTS}

doi:10.1136/ejhpharm-2013-000276.351

$\underline{ }{ }^{1}$ R Ruggiero, ${ }^{2} \mathrm{~A}$ Melillo, ${ }^{2} \mathrm{~L}$ Russo, ${ }^{3} \mathrm{D}$ Micera, ${ }^{4} \mathrm{FA}$ Aliberti, ${ }^{5} \mathrm{G}$ Vighi. ${ }^{\prime} A . O$. "G. Salvini", U.S.C. Farmacia, Rho (MI), Italy; ${ }^{2}$ A.O. "G.Rummo", U.O.C. Farmacia, Benevento, Italy; ${ }^{3}$ A.O. "S. Giuseppe Moscati", U.O.C. Farmacia, Avellino, Italy; ${ }^{4}$ A.O. "S. Giovanni di Dio e Ruggi d'Aragona", U.O.C. Farmacia, Salerno, Italy; ${ }^{5}$ A. O. "Niguarda Ca' Granda", S.S. Qualità e Sicurezza Clinica, Milano, Italy

Background The best polytherapy is associated with a major risk of adverse events (ADEs) and with an increase of both mortality and morbidity.
Purpose To evaluate the frequency of the appearance of ADEs in those patients undergoing polytherapy compared to the frequency of ADEs tied to monotherapy.

Materials and Methods Patients entering A.O 'Gaetano Rummo' of Benevento were monitored by a dedicated hospital pharmacist, over a period of twenty-four months, by collecting data concerning recorded $\mathrm{ADEs}$ and total value analysis (mono/polytherapy), the seriousness and the number of medications considered suspicious.

Results Out of 253 reports made, 140 (55.3\%) involved patients undergoing polytherapy compared to 113 attributable to monotherapy. More precisely, $108 \mathrm{ADEs}$ were considered 'serious' and $55.5 \%$ of these (60 cases) were due to the polytherapy. Out of 48 serious cases imputable to the use of one drug, just 1 has ended with the death of the patient (anaphylactic shock by ceftriaxone), 1 endangered the patient's life and for 16 of them it was remedied by prolonging hospitalisation. Out of 145 cases which were considered by the detector as 'not serious', 80 proved to have been associated with polytherapy while 60 were relative to 1 medicine.

Conclusions The multi-drug approach represents a significant factor which can cause the appearance of ADEs. To improve health care it is desirable that competent professional figures, such as the pharmacist, would more often employed in a departmental activity of pharmacovigilance in order to develop a prior information network on the risk of medicine interactions and the proper use of the medication.

No conflict of interest.

\section{PHC-007 DEPLOYMENT OF BAR CODE MEDICINES ADMINISTRATION TO CONTROL THE ADMINISTRATION OF MEDICINES IN GERIATRIC UNITS}

doi:10.1136/ejhpharm-2013-000276.352

${ }^{1} \mathrm{~F}$ Boye, ${ }^{1} \mathrm{~A}$ Cyrus, ${ }^{1} \mathrm{C}$ Lebaudy, ${ }^{2} \mathrm{P}$ Lambea, ${ }^{3 B}$ Vellas, 'P Cestac. 'University Hospital, Pharmacy, Toulouse, France; 'University Hospital, Computing department, Toulouse, France; ${ }^{3}$ University Hospital, Geriatrics, Toulouse, France

Background Of the errors occurring in drug treatment, about $24 \%$ take place during the step of administration (Mission nationale d'expertise et d'audit hospitaliers (MeaH) 2008). Poon et al, showed in 2010 that the Bar Code Medicines Administration (BCMA) reduced drug administration errors by $41.4 \%$ and serious potential adverse drug events by $54.1 \%$.

Drug prescribing, dispensing and administration have been computerised in the 13 geriatric units at the University Hospital (CHU) of Toulouse. Since January 2012, an additional device has been deployed in 8 wards: barcode readers have been installed to read barcodes on the drug packaging to make administration safer.

Purpose A quality indicator was developed in order to analyse the use of barcode readers in care units in real time, to directly reduce drug administration errors. This indicator is a management tool to ensure that the BCMA system does not deviate over time.

Materials and Methods The indicator was designed with the help of a computer specialist. The request is based on an Access file that extracts administration data from the Disporao prescription software. Two parameters are determined: the number of doses administered by BCMA and the number of administered doses that could be scanned; the ratio of these two elements reflects the use of barcode readers by nurses.

Results The training of 89 nurses was completed in June 2012. The indicator showed that nurses scan an average of $70 \%$ of unit doses. The objective is to scan more than $95 \%$ of unit doses. Investigations are underway to understand the reasons for incompleteness (temporary nursing staff not trained, incorrect prescriptions, faulty hardware, for example) and make corrective actions. 
Conclusions Optimization of the deployment of BCMA in the Geriatric units of Toulouse CHU allows us to plan the development of this practise over a large number of clinical departments at a later date.

No conflict of interest.

\section{PHC-008 DEVELOPMENT AND APPLICATION OF A SIMPLE LC-MS METHOD FOR THE DETERMINATION OF PLASMA RILPIVIRINE CONCENTRATIONS}

doi:10.1136/ejhpharm-2013-000276.353

'M Shibata, 'M Takahashi, 'N Fukushima, 'F Yamaguchi, 'T Nomura, ${ }^{2} Y$ Yokomaku, ${ }^{2}$ W Sugiura. 'National Hospital Organization Nagoya Medical Center, Pharmacy, Nagoya, Japan; ${ }^{2}$ National Hospital Organization Nagoya Medical Center, Clinical Research Center, Nagoya, Japan

Background Rilpivirine is a second-generation non-nucleoside reverse transcriptase inhibitor that is highly potent against both wild-type and drug-resistant HIV-1 strains. The quantification of rilpivirine in human plasma is important to support clinical studies.

Purpose Rilpivirine was just approved in May 2012 in Japan. Therefore, pharmacokinetic studies of rilpivirine have still not been completed in Japanese patients. We intended to develop a conventional method for determining plasma rilpivirine concentrations and compare plasma rilpivirine concentrations of Japanese HIV-1 infected patients with those of foreign healthy volunteers.

Materials and Methods We used a Waters Alliance 2695 HPLC and a Micromass ZQ-2000 MS, controlled with MassLynx version 4.0 software. Our method involves rapid liquid-liquid drug extraction from plasma and use of gradient elution on a reversed-phase C18 column. We recruited 34 Japanese HIV-1 infected patients who were treated with a rilpivirine-containing regimen at the National Hospital Organization Nagoya Medical Center, Japan. All patients had been given $75 \mathrm{mg}$ rilpivirine once daily in combination with other antiretrovirals.

Results The LC-MS method established was validated by estimating the precision and accuracy for inter- and intraday analysis in the concentration range of $18-715 \mathrm{ng} / \mathrm{ml}$. The calibration curve was linear in this range. Average accuracy ranged from 100.0 to $100.6 \%$. Relative standard deviations of both inter- and intraday assays were less than $3.3 \%$. In this study, mean rilpivirine plasma concentration for Japanese patients at trough was $58 \mathrm{ng} / \mathrm{ml}(\mathrm{n}=18)$. Mean rilpivirine concentration at peak was $126 \mathrm{ng} / \mathrm{ml}(\mathrm{n}=6)$. These levels were higher than rilpivirine concentrations seen in trials with healthy foreign volunteers.

Conclusions Our LC-MS method provides a conventional, accurate and precise way of determining rilpivirine in human plasma. In clinical practise, AUC of rilpivirine for Japanese HIV-1 infected patients is larger in comparison with foreign data. We think that this was caused by the poor build of Japanese HIV-1 infected patients.

No conflict of interest.

\section{PHC-009 DRUG DOSE ADJUSTMENT IN RENAL FAILURE}

doi:10.1136/ejhpharm-2013-000276.354

AM Rizo Cerdá, P Selvi-Sabater, MC Sanchez-Mulero, A Boso-Ribelles, B Arribas-Diaz, A Morego-Soler, N Manresa-Ramon, I Sanchez-Martinez, MD Najera-Perez, I SanchezQuilez. Hospital Morales Meseguer, Pharmacy, Murcia, Spain

Background In renal failure, alteration in the pharmacokinetics increases the frequency of overdoses.

Purpose To evaluate pharmaceutical care using a computer programme for drug dose adjustment in renal failure.
Materials and Methods The study period lasted from September 2011 to January 2012 (inclusive), in a 420-bed hospital. Every day creatinine values over $130 \mathrm{mmol} / \mathrm{l}$ were filtered. Treatment was reviewed and we obtained creatinine clearance values (Crockcoft \& Gault) of selected patients. After consulting the drug dose adjustment on the sheet and in Micromedex, a report was sent with the pharmaceutical recommendation.

Results There were 68 interventions for the 2147 patients studied: Internal Medicine (34) Cardiology (1), Short Stay Unit (5), Orthopaedics (7), Urology (5), Haematology (7) Surgery (5), Neurology (1), Intensive Care Unit (ICU) (2) Oncology (1). 55.9\% of notifications were for changes in the dose of enoxaparin (38), $11.8 \%$ of amoxicillin-clavulanic acid (8), piperacillin-tazobactam 14.7\% (10), $8.8 \%$ levofloxacin (6), 2.9\% meropenem (2), 2.9\% ciprofloxacin (2), $1.5 \%$ imipenem (1) and $1.5 \%$ aztreonam (1). The proportion of suggested changes accepted was $58.8 \%$ (40). $5.9 \%$ (4) discontinued treatment, $5.9 \%$ (4) were discharged and $29.4 \%$ (20) not changed. Of the latter, five were for changes in the pattern of enoxaparin in trauma patients, another 5 from Internal Medicine and 2 more from Haematology and ICU. The rest of them were changes in the pattern of antibiotics (imipenem 1, 2 levofloxacin, 1 meropenem, 1 ciprofloxacin, piperacillin-tazobactam 3) that were given out in the different services.

Conclusions A high percentage of doctors followed the recommendations. Part of the unaccepted tally corresponds to trauma patients whose prophylactic regimen of enoxaparin $(40 \mathrm{mg} / 24 \mathrm{~h}$ ) was not modified due to the service criteria. Some of the antibiotic prescriptions were not changed because of the severity of the patient's illness (1 levofloxacin and 1 Internal Medicine Meropenem Imipenem Oncology and 1). The rest were rejected without explanation.

No conflict of interest.

\section{PHC-010 DRUG INTERACTION: A CASE REPORT}

doi:10.1136/ejhpharm-2013-000276.355

E Fernández López, E Tevar Alfonso, MA Ocaña Gomez, JA De León Gil, I Plasencia García, J Merino Alonso, R Jurado López, E Marqués Guell. Hospital Nuestra Señora de Candelaria, Farmacia Hospitalaria, Santa Cruz de Tenerife, Spain

Background The serum concentration of valproic acid (VPA) in epilepsy patients is reduced to sub-therapeutic by the administration of carbapenems antibiotics.

Purpose Description of the interaction and communication to the Pharmacovigilance Center with yellow cards.

Materials and Methods A 66-year-old was admitted to the resuscitation unit after being operated on for perforation peritonitis secondary to cytomegalovirus. Treatment was with imipenem because the suspicion of extended-spectrum beta-lactamases (ESLB) organisms was confirmed. Concomitant treatment was with VPA $400 \mathrm{mg}-400 \mathrm{mg}-400 \mathrm{mg}$ due to an underlying disease, epilepsy. The pharmacy department was asked to cheque the VPA blood level: initially levels were within the therapeutic interval (TI), but at 24 hours after starting treatment with imipenem it decreased by $70 \%$ to below the TI. In addition, because of the proconvulsive properties of imipenem, the patient started to have convulsions.

After reporting the suspected interaction, the doctor decided to change the antibiotic to meropenem $1 \mathrm{~g} / 8 \mathrm{~h}$ and so eliminate at least the pharmacodynamic component of the interaction. After 24 hours of the change VPA levels continued to fall and at 48 hours were almost undetectable $(\leq 3 \mathrm{mcg} / \mathrm{mL})$. VPA dose was increased, 1000 mg-1200 mg-1000 mg, without the situation reversimg. After 30 days meropenem was suspended and VPA levels did not return to the TI until after approximately $120 \mathrm{~h}$.

Results Although the exact mechanism is unknown, it is suspected to be of the pharmacokinetic kind and at several levels: 